\title{
Joint Dynamic Strategy of Bayesian Regularized Back Propagation Neural Network with Strong Robustness - Extended Kalman Filtering for the Battery State-of-Charge Prediction
}

\author{
Yifen Hu$u^{1}$ Yixing Zhang ${ }^{2}$ Shunli Wang $^{2} \cdot$ Wenhua Xu ${ }^{2} \cdot$ Yongcun Fan2·Yuyang Liu ${ }^{2}$ \\ ${ }^{1}$ School of Automotive and Mechanical-electronic Engineering, Xinyang Vocational and Technical \\ College, Xinyang 464000, China \\ ${ }^{2}$ School of Information Engineering, Southwest University of Science and Technology, Mianyang, \\ 621010, China \\ *E-mail: 497420789@qq.com
}

Received: 3 July 2021 / Accepted: 25 August 2021 / Published: 10 October 2021

\begin{abstract}
Accurate estimation of the state of charge plays an important role in real-time monitoring and safety control of lithium-ion batteries. In practical application, the use of lithium-ion battery will face different sudden noise. Extended Kalman filtering (EKF) is deficient in this kind of processing, so this paper combines EKF with Bayesian regularized backpropagation neural network, and uses dynamic strategy to implement two algorithms to improve the accuracy and speed. Experimental results show that the joint algorithm has a stable effect and a good tracking effect under sudden noise conditions. Compared with the extended Kalman filtering algorithm, the average error of the algorithm in the capacity test is reduced by $0.797 \%$, and the maximum error is reduced by $2.651 \%$. In the dynamic stress test and the pulse test, the average error was reduced by $0.2683 \%$ and $0.3919 \%$, and the maximum error was reduced by $7.195 \%$ and $7.769 \%$, respectively. It is verified that the algorithm combining the extended Kalman filtering and the back propagation neural network has high accuracy in the estimation of the state of charge of the lithium-ion battery under sudden events.
\end{abstract}

Keywords: Sudden noise; Dynamic programming; BP neural network; Real-time; Bayesian regularized;

\section{$\underline{\text { FULL TEXT }}$}

(C) 2021 The Authors. Published by ESG (www.electrochemsci.org). This article is an open access article distributed under the terms and conditions of the Creative Commons Attribution license (http://creativecommons.org/licenses/by/4.0/). 